\title{
Toward a Flexible and Portable CT Scanner
}

\author{
Jeff Orchard ${ }^{1}$ and John T.W. Yeow ${ }^{2}$ \\ ${ }^{1}$ David R. Cheriton School of Computer Science, University of Waterloo, Canada \\ jorchard@uwaterloo.ca \\ 2 Systems Design Engineering, University of Waterloo, Canada
}

\begin{abstract}
The very hot and power-hungry x-ray filaments in today's computed tomography (CT) scanners constrain their design to be big and stationary. What if we built a CT scanner that could be deployed at the scene of a car accident to acquire tomographic images before moving the victim? Recent developments in nanotechnology have shown that carbon nanotubes can produce x-rays at room temperature, and with relatively low power needs. We propose a design for a portable and flexible CT scanner made up of an addressable array of tiny x-ray emitters and detectors. In this paper, we outline a basic design, propose a strategy for reconstruction, and demonstrate the concept using a software simulation of the scanner. We also raise a number of issues that still need to be overcome to build such a scanner.
\end{abstract}

Keywords: computed tomography, x-ray, reconstruction, carbon nanotubes.

\section{Introduction}

Current clinical computed tomography (CT) scanners are rather large and therefore stationary. Their basic design includes a single $\mathrm{x}$-ray source that traverses a circular trajectory around the patient, and detectors on the opposite side of the patient sense the transmitted x-rays. The x-ray source requires a lot of power and is very hot, contributing to the reason why CT scanners are designed to be stationary.

Recently, carbon nanotubes (CNTs) have been used to generate x-rays at room temperature, consuming far less power than x-ray emitters in current $\mathrm{CT}$ scanners 1. Moreover, work is being done to integrate these tiny x-ray devices into a flexible array such that each emitter can be individually pulsed [2]. While such an "x-ray blanket" could have many applications, we focus here on the concept of using the array, in conjunction with x-ray detectors, as a flexible and portable CT scanner. In this paper, we outline a design concept for a flexible CT scanner, and use computer simulations to demonstrate the feasibility of using the data from the scanner to reconstruct tomographic images, despite the scanner's irregular geometry. At the conclusion, we outline several hurdles that must be overcome in order to build a working prototype of the scanner.

D. Metaxas et al. (Eds.): MICCAI 2008, Part II, LNCS 5242, pp. 188 195, 2008.

(C) Springer-Verlag Berlin Heidelberg 2008 


\section{Background}

Carbon nanotubes belong to the family of fullerene structures. Since their discovery by Iijima in 1991, research into the science and applications of CNTs has been relentless [3. One interesting property of CNTs is field emission, the ability to emit electrons from their tips when they are subject to an electric field. Field emission from CNTs was first reported in 1995 by Rinzler et al. 44, de Heer et al. 5], and Chernozatonskii et al. 6]. The high aspect ratio of the CNTs serves to amplify the electric field. Together with a small work function of 4 to $5 \mathrm{eV}$ 7], CNTs have shown to be excellent field emitters. When exposed to an electric field in ultra high vacuum, each strand of CNT will emit electrons spontaneously. Kinetic energy is imparted on the free electrons by attracting them with a high voltage anode. X-rays are generated when the high energy electrons hit the heavy metal anode.

The advent of CNT-based x-ray generators has opened the possibility of miniature x-ray sources. Experiments by Zhang et al. [18] have produced x-ray images from a small bank of CNT emitters, each spaced just over a centimetre apart. Smaller x-ray generators could potentially be interleaved with detectors around the patients. In this architecture, an active subset of x-ray generators would produce multiple fan beams from different angles. Each x-ray generator is digitally addressable and can be multiplexed to mimic the mechanical rotation of a CT gantry system. Moreover, electronic switching will allow multiple x-ray images to be acquired using the modulation/demodulation technique, as proposed by Zhang et al. 8].

Carbon nanotubes have recently been proposed for CT scanners. Quan et al. 9] proposed two designs for CT scanners based on pulsed CNT emitters. Their designs include square and hexagonal arrangements of flat-panel emitter and detector arrays positioned across from one another. The emitters are pulsed, one at a time, and the entire set of detector arrays is sampled for each emitter pulse. They used computer simulations to demonstrate the feasibility of reconstructing images using these geometries.

While these designs are an interesting departure from the standard clinical CT scanner, none are proposed as a flexible or portable device. Here, we focus on a design concept that is intended to be versatile, flexible and portable.

\section{Outline of the Scanner}

The general design for our new breed of CT scanners is to embed an array of CNT x-ray emitters and silicon photodiode detectors into a flexible sheet and wrap it around an object (as shown in Fig. 1) to collect x-ray projections. These projections, when combined with positional information for the scanner, can be used to reconstruct CT images. Here, we describe a specific prototype that we have implemented as a software simulation.

The (virtual) emitters and detectors are interleaved on a rectangular, flexible sheet that can be wrapped around an object or body part to form a cylindrical 


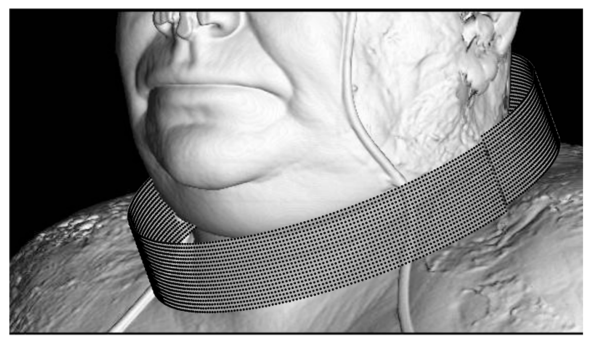

(a) 3-D Rendering

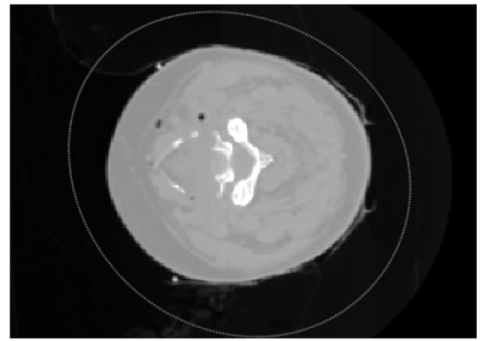

(b) Cross-Section

Fig. 1. Flexible CT scanner around the neck. The 3-D rendering was done using the VolumeJ plugin [10] for ImageJ [1].

(tube-like) shape. To be comparable to current fixed CT scanners, the emitters and detectors are spaced so that they are approximately $1^{\circ}$ apart when in a cylindrical configuration. The flexible sheet contains a $360 \times 19$ grid of interleaved emitters and detectors forming a checker-board pattern. If the devices are spaced $2 \mathrm{~mm}$ apart, the total circumference of the scanner is $72 \mathrm{~cm}$, yielding a diameter of $23 \mathrm{~cm}$. In total, we have 3420 emitters and 3420 detectors. This geometry is similar to that of a multiring positron emission tomography (PET) scanner, and hence shares some of the same reconstruction methods.

Unlike most CT scanners, however, our scanner does not have a fixed circular geometry. Since the flexible nature of the scanner means that each scan might involve a different geometry, our reconstruction method is forced to think of the scan as a collection of individual ray sums without any a priori geometrical relationship. Rather the geometry of the scanner is dictated by a positional measurement system. This makes reconstruction more challenging.

Before diving into the general-geometry 3-D reconstruction, let us first consider the case where the scanner is perfectly cylindrical. The x-ray transform for a 3-D scanner is 4-dimensional; each ray has two endpoints, and each endpoint can be specified by two coordinates within the scanner. Figure 2 depicts a single ray in a $3-\mathrm{D}$ cylindrical scanner, where the ray travels from $\mathbf{A}$ to $\mathbf{B}$. The figure shows a common parameterization of this 4-D space, $(s, \phi, z, \delta)[12$. Briefly, $s$ and $\phi$ correspond to the polar coordinates of the ray when projected onto a transaxial plane, $z$ is the average of the $z$-values for $\mathbf{A}$ and $\mathbf{B}$ (i.e. the $z$-value midpoint), and $\delta$ represents the tangent of the angle between the transaxial plane and the plane containing points $\mathbf{A}, \mathbf{B}$ and $(0,0, z)$.

Since our 3-D object is represented by 4-D data, it stands to reason that the 4-D representation contains redundancy. The FORE-J method [13] is a Fourier rebinning method (used to reconstruct PET images) that combines the redundant data of multiple oblique sinograms into a set of transaxial sinograms that can then be reconstructed independently using a standard 2-D parallel-beam method. The redundancy is exploited to improve the signal-to-noise ratio (SNR) within the transaxial sinograms. Other PET reconstruction methods have been developed to leverage the redundant data to improve SNR 1415 16] (see [17] 


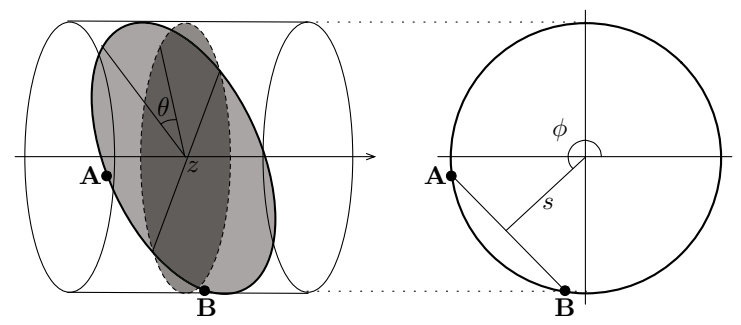

Fig. 2. Geometry of the $(s, \phi, z, \delta)$ x-ray transform space, where $\delta=\tan \theta$. The scanner $z$-axis runs along the cylinder's axis of symmetry.

and [18] for surveys). Fourier rebinning methods are advantageous because of their efficiency. An advantage of the FORE-J method, in particular, is that it requires interpolation along only one dimension during resampling of the 4-D x-ray transform space.

As we relax the constraint that the scanner be cylindrical, we still assume that the position of each device (emitter and detector) is known (more on this in the Conclusions). The positions of three particular emitters on the scanner are used to define a scanner-fixed reference frame. The origin of the reference frame is at the scanner's centroid and the three emitters are used in concert to define the directions of the coordinate-system axes. Transaxial sinograms are defined to be perpendicular to the $z$-axis of this coordinate system.

\section{Simulations}

This paper acts as a proof-of-concept for the feasibility of reconstructing meaningful images from a flexible CT scanner. As such, the physical device does not yet exist. Instead, the experiments done in this study are based on data generated using a software simulator of the flexible CT scanner.

Simulation of the virtual CT scanner starts with the sheet of emitters and detectors rolled into a perfectly cylindrical tube such that the longer rows of 360 devices form rings, and the shorter columns of 19 devices form rows that run the length of the cylinder. The cone beam of each x-ray emitter has a apex half-angle of $60^{\circ}$, and the centre of the beam passes through the scanner's $z$-axis. We denote this configuration as the baseline.

The baseline geometry is then deformed using a bivariate cubic spline defined by a grid of 20 control points. Hence, by moving the control points of the displacement grid, we can generate different smoothly-varying geometrical configurations (see Fig. 1(b)). The direction of each emitter's x-ray cone is determined using the normal vector of the spline surface.

In our simulations, we do not incorporate the angle of incidence into the detector response. Since our method assumes we know the geometry, the angle of incidence can be computed and any directional effect can be compensated for. 


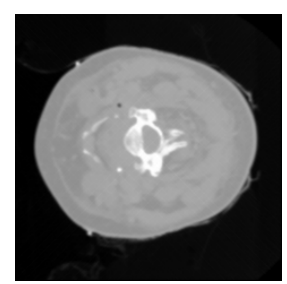

(a) Circular

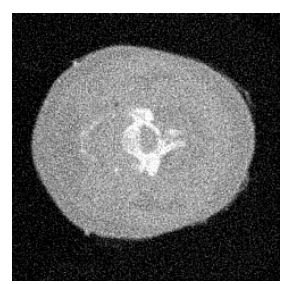

(b) Circular, noisy

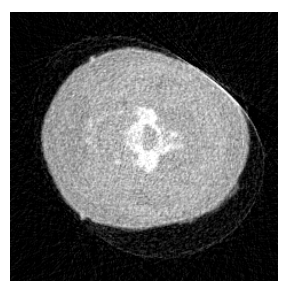

(c) Non-FORE-J

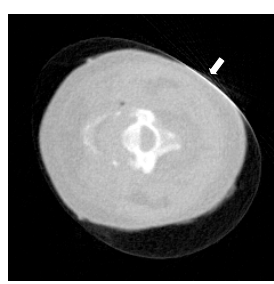

(d) FORE-J

Fig. 3. Sample reconstructions. (a) is a control image from a circular 2-D scan. (b) is the same as (a), but with $5 \%$ Gaussian noise. (c) and (d) show the corresponding reconstructions generated using the data from the flexible scanner, including $5 \%$ noise. The arrow in $(\mathrm{d})$ points to an edge artifact.

To demonstrate the reconstruction quality for this irregular geometry, we use a $236 \times 330 \times 105$ voxel $\mathrm{CT}$ volume (approx. $1 \mathrm{~mm}$ voxels) of the neck from the Visible Female dataset (Visible Human Project, National Library of Medicine). As shown in Fig. 1] the virtual CT scanner is positioned around the neck, and Xray projections are computed by evaluating the line integrals along the straight lines from each emitter to each detector in the emitter's x-ray footprint. The integrals are approximated by taking steps of size $0.3 \mathrm{~mm}$ and interpolating the volume intensity using trilinear interpolation.

The CT data generated from the above simulator is fed into a reconstruction method that involves resampling the irregular projections onto a regular grid in the 4-D x-ray transform space. To accomplish this, the maximum extents for each of the four parameters is determined and the ranges are partitioned into bins: 360 for the $s$ axis, 180 for the $\phi$ axis, 19 for the $z$ axis, and 10 for the $\delta$ axis. The influence of each ray sum is distributed linearly onto its 16 nearest samples. The final value for a sample is the weighted average of the contributing ray sums (undefined samples are handled within the FORE-J method [13]).

Once the data is on a regular grid, we use the FORE-J method to reconstruct the images [13. Figure 3 shows four reconstruction examples. Two of the images ((a) and (b)) are reconstructions from a Radon transform that was populated using the traditional circular scanner geometry; the Radon transforms were created using Matlab's (MathWorks Inc., Natick, Massachusetts) radon function, and the slice reconstructions were done using Matlab's iradon function. Image (a) is almost indistinguishable from the original slice (not shown), while (b) was reconstructed from CT data that was artificially corrupted by adding Gaussian distributed noise to each ray sum (standard deviation of $5 \%$ of average ray sum).

The images shown in Figs. 3(c) and (d) were reconstructed from the simulated data acquired from the flexible CT scanner depicted in Fig. 1 The ray sums were also artificially corrupted with 5\% additive Gaussian noise. Image (c) was reconstructed from the rebinned transaxial sinograms only, essentially throwing away all the oblique sinogram data. Image (d) was reconstructed using the FORE-J algorithm [13, and shows considerably less noise than (b) or (c) because it uses the redundant data in the oblique sinograms to improve the SNR. 
Figures 3(c) and (d) exhibit an edge artifact, indicated by the arrow in (d). In our simulations, we assume that an emitter's x-ray beam forms a cone with an apex angle of $120^{\circ}$ (deviating $60^{\circ}$ from the emitter's central view line). This wide-angle cone-beam is needed to capture the required data. If the angle was smaller, or if the CT scanner was placed closer to the skin of the patient, then some non-zero ray sums would fail to be acquired. Comparing Figs. 1) (b) and 3)(d), one can see that the closest approach of the scanning device to the body results in a slight loss of information, and ultimately in an edge artifact.

\section{Future Challenges}

There are many challenges that must be overcome before a flexible and portable CT scanner can be built. In this section, we outline the most obvious issues.

Each CNT has to be in a vacuum for it to emit x-rays. The prospect of encasing each emitter in its own vacuum container poses a forbidding technical challenge. Less daunting is the idea of forming small squares, perhaps $2 \mathrm{~cm}$ on a side, housing several emitters and detectors. These plates could be hinged together to form an approximation to the flexible scanner outlined in this paper. In fact, sensors could be used to measure the angles between the plates as a means for motion tracking.

The structural integrity of carbon nanotubes degrades over time. This degradation results in reduced x-ray flux, and hence factors prominently in the use of CNTs for our application. Moreover, the nature of the degradation processes are not well understood. More research is needed to address this issue.

It is not clear how much x-ray photon flux we can expect from CNTs. Physical experiments have produced some compelling results, with exposure times on the order of 10 seconds [18. If the flux is too low, then the image quality will suffer with a low SNR. This low flux issue can be mitigated by longer scan times, but then patient motion becomes more of a problem.

As x-ray photons pass through the tissues of the body, a percentage of them undergo Rayleigh and Compton scattering [19. As a result, a razor-sharp x-ray beam gets blurred before it reaches the detector. All CT scanners suffer from this phenomenon. We have not studied the effects of scatter in the context of our flexible scanner. However, we anticipate that the effects of scattering will be similar to those in other forms of CT scans. Further experiments are needed to confirm this hypothesis.

The geometrical conformation of the scanner sheet (the positions of the emitters and detectors) is an essential input to the reconstruction software outlined in this paper. ShapeTape, a motion-tracking device designed to track the curves traced by a thin strip in 3D, seems like an obvious choice for this application. While ShapeTape is designed to report shape in a high-dimensional space, its ability to be used as an absolute positioning system is problematic 20. However, one can imagine that a second motion-tracking system could be used to augment the shortcomings of the ShapeTape. For example, a grid of motiontracking markers on the scanner sheet could give a good absolute position, while 
the ShapeTape acts like an interpolator, giving information about how the sheet bends between the markers. The absolute motion-tracking system probably cannot be line-of-sight based (like an optical motion-tracking system), so is more likely to be an electromagnetic tracking system. However, these tracking systems can experience interference in the presence of metal objects, which might pose a problem for many scenarios involving traffic accidents. Another option to measure scanner geometry is to use radio-frequency (RF) ranging. This technology is similar to the global positioning system (GPS), except that our system would have to use higher frequencies, and our synchronization would be easier because the sensors could be connected by wires.

The effect of errors in reconstruction geometry were shown to be well-behaved in a 2-D study 21. Moreover, a study based on the simulation of a flexible single-slice scanner demonstrated that geometrical errors could be corrected by fine-tuning the scanner geometry to minimize the image entropy [22]. A 3-D implementation of the idea, while workable in principle, would involve a more challenging and computationally expensive optimization problem. We plan to conduct more studies on the impact of geometrical errors.

Finally, there will certainly be safety issues with an x-ray device that can be deployed outside of a controlled environment. Obviously, measures need to be taken to prevent $\mathrm{x}$-ray exposure to those who deploy and operate the scanners.

\section{References}

1. Zhang, J., Yang, G., Cheng, Y., Gao, B., Qiu, Q., Lee, Y.Z., Lu, J.P., Zhou, O.: Stationary scanning $\mathrm{X}$-ray source based on carbon nanotube field emitters. Applied Physics Letters 86(184104) (2005)

2. Sinha, N., Yeow, J.T.W.: Carbon nanotubes for biomedical applications. IEEE Transactions on Nanobioscience 4(3), 180-195 (2005)

3. Iijima, S.: Helical microtubules of graphitic carbon. Nature 354, 56-58 (1991)

4. Rinzler, A.G., Hafner, J.H., Nikolaev, P., Nordlander, P., Colbert, D.T., Smalley, R.E., Lou, L., Kim, S.G., Tománek, D.: Unraveling nanotubes: Field emission from an atomic wire. Science 269(5230), 1550-1553 (1995)

5. de Heer, W.A., Châtelain, A., Ugarte, D.: A carbon nanotube field-emission electron source. Science 270(5239), 1179-1180 (1995)

6. Chernozatonskii, L.A., Gulyaev, Y.V., Kosakovskaja, Z.J., Sinitsyn, N.I., Torgashov, G.V., Zakharchenko, Y.F., Fedorov, E.A., Val'chuk, V.P.: Electron field emission from nanofilament carbon films. Chemical Physics Letters 233, 63-68 (1995)

7. Sun, J., Zhang, Z., Hou, S., Zhang, G., Gu, Z., Zhao, X., Liu, W., Xue, Z.: Work function of single-walled carbon nanotubes determined by field emission microscopy. Applied Physics A 75(4), 479-483 (2002)

8. Zhang, J., Yang, G., Lee, Y.Z., Chang, S., Lu, J.P., Zhou, O.: Multiplexing radiography using a carbon nanotube based x-ray source. Applied Physics Letters 89(064106) (September 2006)

9. Quan, E., Lalush, D.S.: Evaluation of hexgonal and square geometries for motionfree arrayed-source x-ray micro-CT. In: Proc.of the IEEE International Symposium of Biomedical Imaging (ISBI 2007), pp. 221-224 (2007) 
10. Abramoff, M.D., Viergever, M.A.: Computation and visualization of three dimensional motion in the orbit. IEEE Trans. Med. Imag. 21(4) (2002)

11. Abramoff, M.D., Magelhaes, P.J., Ram, S.J.: Image processing with Image. J. Biophotonics International 11(7), 36-42 (2004)

12. Defrise, M., Kinahan, P.E., Townsend, D.W.: Exact and approximate rebinning algorithms for 3-D PET data. IEEE Trans. Med. Imag. 16(2), 145-158 (1997)

13. Defrise, M., Liu, X.: A fast rebinning algorithm for 3D positron emission tomography using John's equation. Inverse Problems 15(4), 1047-1065 (1999)

14. Cho, Z.H., Ra, J.B., Hilal, S.K.: True three-dimensional reconstruction (TTR) application of algorithm toward full utilization of oblique rays. IEEE Trans. Med. Imag. MI-2(1), 6-18 (1983)

15. Kinahan, P.E., Rogers, J.G.: Analytic 3D image reconstruction using all detected events. IEEE Transactions on Nuclear Science 36(1), 964-968 (1989)

16. Lewitt, R.M., Muehllehner, G., Karp, J.S.: Three-dimensional image reconstruction for PET by multi-slice rebinning and axial image filtering. Physics in Medicine and Biology 39(3), 321-339 (1994)

17. Defrise, M., Geissbuhler, A., Townsend, D.W.: A performance study of 3D reconstruction algorithms for positron emission tomography. Physics in Medicine and Biology 39(3), 305-320 (1994)

18. Baghaei, H., Wong, W.H., Uribe, J., Wang, Y., Liu, Y., Xing, T., Ramirez, R., Xie, S., Kim, S.: A comparison of four image reconstruction algorithms for detection of small lesions in brain phantom. In: Nuclear Science Symposium Conference Record, vol. 4, pp. 2584-2588 (October 2003)

19. Hsieh, J.: Computed Tomography: Principles, Design, Artifacts, and Recent Advances. SPIE Press, Bellingham (2003)

20. Baillot, Y., Eliason, J.J., Schmidt, G.S., Swan, I.J.E., Brown, D., Julier, S., Livingston, M.A., Rosenblum, L.: Evaluation of the ShapeTape tracker for wearable, mobile interaction. In: Proc. of the IEEE Virtual Reality (2003)

21. Ramotar, A., Orchard, J.: General geometry CT reconstruction. In: Arabnia, H.R. (ed.) Proc. of the International Conference on Image Processing and Computer Vision (IPCV 2006), vol. 1, pp. 95-99 (June 2006)

22. Orchard, J., Ramotar, A.: Autocorrecting reconstruction for flexible CT scanners. In: Proc. of the IEEE International Symposium of Biomedical Imaging (ISBI 2007), pp. 804-807 (April 2007) 